\title{
Primary Care Physicians' Perspective on Pharmacists Delivering Vaccines to Adults
}

\author{
Christine E. MacBrayne, PharmD, MSCS, Laura P. Hurley, MD, MPH, \\ Sean T. O'Leary, MD, MPH, Jessica R. Cataldi, MD, MSCS, Lori A. Crane, PhD, MPH, \\ Carol Gorman, BA, Michaela Brtnikova, PhD, MPH, Brenda L. Beaty, MSPH, and \\ Allison Kempe, MD, MPH
}

Introduction: Since 2009, pharmacists in all 50 states have been authorized to provide vaccinations to adults. The objective of this study was to assess primary care physicians' (PCPs) experiences with and attitudes about pharmacists administering vaccinations.

Methods: Internet and mail survey of PCPs representative of American College of Physicians' and American Academy of Family Physicians' memberships.

Results: Response rate was 69\% (642/926). Ninety-eight percent of respondents agreed (79\% "Strongly," 19\% "Somewhat") that it is their responsibility to assure their adult patients receive recommended vaccinations. Most respondents agreed that pharmacists either did not have access to patient medical information (33\% "Strongly," $45 \%$ "Somewhat") or did not have adequate vaccination history (33\% "Strongly," 41\% "Somewhat"). The majority also agreed that pharmacists did not inform them when vaccinations were given (35\% "Strongly," 39\% "Somewhat") and did not enter vaccinations administered into immunization information systems (IISs) (20\% "Strongly," 37\% "Somewhat"). However, $83 \%$ agreed (31\% "Strongly," $52 \%$ "Somewhat") that it is helpful to have pharmacists share the role of vaccinating adults.

Conclusions: PCPs have mixed feelings about pharmacists delivering vaccines. Universal use of IISs by pharmacists could partially address physicians' concerns by providing a systematic way for pharmacists and physicians to share patient vaccination histories. (J Am Board Fam Med 2021;34:392-397.)

Keywords: Immunization, Pharmacists, Primary Care Physicians, Primary Health Care, Surveys and Questionnaires, Vaccines

\section{Introduction}

Adult vaccinations have proven effective at preventing morbidity and mortality as well as protecting the community from vaccine-preventable diseases, yet vaccines for adults are underused. ${ }^{1}$ Vaccinations

This article was externally peer reviewed.

Submitted 17 September 2020; revised 6 November 2020; accepted 11 November 2020.

From the Department of Pharmacy, Children's Hospital Colorado, Aurora, (CEM); Adult and Child Consortium for Health Outcomes Research and Delivery Science, University of Colorado Anschutz Medical Campus and Children's Hospital Colorado, Aurora (LPH, STO, JRC, LAC, CG, MB, BLB, AK); Division of General Internal Medicine, Denver Health, Denver, CO (LPH); Department of Pediatrics, University of Colorado Anschutz Medical Campus, Aurora (STO, JRC, MB, AK); Department of Community and Behavioral Health, Colorado School of Public Health, University of Colorado Anschutz Medical Campus, Aurora (LAC).

Funding: Centers for Disease Control and Prevention, cooperative agreement 1U01IP000849-02. have traditionally been delivered in physician practices, hospitals, and public health systems. Pharmacists administering vaccinations has been a rapidly growing practice since the 1990s. Since 2009, pharmacists in all 50 states have been authorized to provide vaccinations, and during the 2014 to 2015 influenza season, 22\% of adults received a vaccination at a retail pharmacy. ${ }^{2-4}$

As more pharmacists deliver vaccinations, there has been increased discussion surrounding the pharmacist's

Conflict of interest: None declared.

Disclaimer: The findings and conclusions in this report are those of the authors and do not necessarily represent the official position of the Centers for Disease Control and Prevention.

Corresponding author: Christine E. MacBrayne, PharmD, MSCS, Children's Hospital Colorado-Department of Pharmacy, 13123 E 16th Ave-Box 375, Aurora, CO 80045 (E-mail: Christine.macbrayne@childrenscolorad.org). 
role in administering vaccinations, including dialog around how receipt of a vaccination in a pharmacy would affect the patient's "medical home." In 2007, the "Joint Principles of the Patient-Centered Medical Home" was issued, outlining an approach to provide comprehensive primary care for children, youth, and adults. These principles outline the importance of the use of information technology and communication for coordinated care across all disciplines within the complex health care system. ${ }^{5}$ To address the concerns of how vaccinations received in a pharmacy would affect the integrity of the patient's "medical home," the National Vaccine Advisory Committee (NVAC) published "Standards for Adult Immunization Practice" that highlighted the critical role that all vaccine providers have in improving vaccination coverage as well as documenting receipt of vaccination. ${ }^{6}$ The American Pharmacist Association coined the term "immunization neighborhood" outlining a collaborative approach to providing vaccinations. ${ }^{4,6,7}$ Pharmacists have an important role in providing vaccinations to adults due to several vaccines being covered by Medicare Part D, a pharmaceutical benefit; therefore, they can play a large role in establishing an immunization neighborhood. Thus, understanding primary care physicians' (PCPs) perceptions and experience with pharmacists administering vaccinations is a fundamental next step for furthering this relationship. ${ }^{7}$

This study aimed to assess PCPs' experience with and attitudes about pharmacists administering vaccinations to their adult patients.

\section{Methods}

\section{Study Setting}

From January to February 2019, we administered a survey to physicians who spent at least half their time practicing primary care. The survey was conducted by the Vaccine Policy Collaborative Initiative, a survey effort that assesses physician attitudes about vaccine issues in collaboration with the Centers for Disease Control and Prevention (CDC). We established a network of PCPs by recruiting general internal medicine physicians (GIMs) and family practice physicians (FPs) from the membership of the American College of Physicians (ACP) and the American Academy of Family Physicians (AAFP). We performed quota sampling to ensure that networks of physicians were comparable to the ACP and AAFP memberships with respect to practice setting, region, and urban versus rural location. ${ }^{8,9}$

\section{Survey Design}

We used 4-point Likert scales for assessing physician agreement with statements about delivery of vaccinations to adult patients by pharmacists ("Strongly Agree" to "Strongly Disagree"). A national advisory panel of GIMs $(n=6)$ and FPs $(\mathrm{n}=5)$ pretested the survey, which was modified based on their feedback. We pilot tested the survey among 71 GIMs and 13 FPs nationally and further modified the survey instrument based on their feedback.

\section{Survey Administration}

Depending on individual physician preference, the survey was administered through US mail or the Internet (using Qualtrics). We sent the mail group an initial mailing and up to 2 additional mail reminders and the Internet group an initial e-mail with up to 8 e-mail reminders. Nonrespondents in the Internet group were also sent up to 2 mail surveys in case of problems with e-mail communication reminders. ${ }^{\text {? }}$

\section{Statistical Analysis}

We pooled Internet and mail surveys for analyses. ${ }^{8,10-12}$ We compared characteristics of respondents with nonrespondents and responses by type of practitioner (GIM or FP) and practice setting (private practice, HMO, hospital/clinic) using $t$ test for continuous variables and Pearsons's $c^{2}$ or Fisher's exact tests for categorical variables, as appropriate. Characteristics of nonrespondents were obtained from the recruitment survey for the sentinel networks. All analyses were performed using SAS, version 9.4 (SAS Institute, Cary, NC).

\section{Results}

The overall response rate was $69 \%$ (642/926), with GIM response rate of $74 \%(364 / 494)$ and FP response rate of $64 \%$ (278/432). Respondents were on average younger and had a larger median number of providers in their practice. Respondents did not differ from nonrespondents by other practice and physical characteristics (Table 1).

Ninety-eight percent of respondents agreed (79\% "Strongly," 19\% "Somewhat") that it is their responsibility to see that their adult patients receive recommended vaccines even if they get them outside of the practice. Most respondents had concerns that pharmacists either did not have access to 
Table 1. Comparison of Characteristics of Responders and Nonresponders ( $(\mathrm{n}=926)$

\begin{tabular}{|c|c|c|c|c|}
\hline Characteristic & Category & $\begin{array}{c}\text { Nonresponder Col \% (n) } \\
\text { N = } 284\end{array}$ & $\begin{array}{c}\text { Responder Col \% (n) } \\
\mathrm{N}=642\end{array}$ & $P$ value \\
\hline \multirow[t]{2}{*}{ Gender } & Female & $41.0 \%(116)$ & $45.7 \%(293)$ & \multirow[t]{2}{*}{0.18} \\
\hline & Male & $59.0 \%(167)$ & $54.3 \%(348)$ & \\
\hline \multirow[t]{3}{*}{ Practice setting } & Hospital or clinic & $16.5 \%(47)$ & $20.2 \%(130)$ & \multirow[t]{3}{*}{0.37} \\
\hline & $\mathrm{HMO}$ & $4.9 \%(14)$ & $5.5 \%(35)$ & \\
\hline & Private practice & $78.5 \%(223)$ & $74.3 \%(477)$ & \\
\hline \multirow[t]{3}{*}{ Census location } & Rural & $10.3 \%(29)$ & $9.7 \%(62)$ & \multirow[t]{3}{*}{0.05} \\
\hline & Urban-non-inner & $59.4 \%(167)$ & $67.3 \%(429)$ & \\
\hline & Urban-inner & $30.2 \%(85)$ & $22.9 \%(146)$ & \\
\hline \multirow[t]{4}{*}{ Region of country } & Midwest & $26.1 \%(74)$ & $25.5 \%(164)$ & \multirow[t]{4}{*}{0.98} \\
\hline & Northeast & $21.1 \%(60)$ & $20.4 \%(131)$ & \\
\hline & South & $31.7 \%(90)$ & $31.9 \%(205)$ & \\
\hline & West & $21.1 \%(60)$ & $22.1 \%(142)$ & \\
\hline \multirow[t]{2}{*}{ Decision-making } & Independent & $58.0 \%(163)$ & $57.4 \%(366)$ & \multirow[t]{2}{*}{0.86} \\
\hline & Larger system level & $42.0 \%(118)$ & $42.6 \%(272)$ & \\
\hline Mean $(\mathrm{SD}) /$ median age in years & & $56.5(9.0) / 58$ & $53.9(9.5) / 54$ & $<0.0001$ \\
\hline Mean (SD)/median number of providers & & $9.5(20.5) / 5$ & $14.6(83.6) / 6$ & $0.03 *$ \\
\hline
\end{tabular}

${ }^{*}$ Wilcoxon test.

$\mathrm{HMO}$, health maintenance organization; SD, standard deviation.

patient medical information (33\% "Strongly," 45\% "Somewhat") or did not have adequate vaccination history (33\% "Strongly," $41 \%$ "Somewhat") to determine if an adult needs a vaccination, with more GIMs strongly agreeing that pharmacists do not have access to patient medical information than FPs $(P=.005)$. The majority also reported that pharmacists did not inform them when vaccines are given (35\% "Strongly," 39\% "Somewhat") and did not enter vaccines administered into immunization information systems (IISs) (20\% "Strongly," 37\% "Somewhat") (Figure 1). There were no other differences in responses between type of practitioner or practice setting.

\section{Discussion}

This study found that a high proportion of physicians had concerns that pharmacists do not have access to adequate patient medical information or vaccination history to determine if an adult needs a vaccination, that pharmacists do not inform them when vaccinations are given, and that they do not enter vaccinations administered into an IIS. These findings suggest that collaboration between PCPs and pharmacists needs improvement. Despite these concerns, a large majority of respondents felt that it is helpful to have pharmacists share the role of vaccinating adults with physicians, and 50\% agreed that it is more convenient for adults to get vaccinations at a pharmacy than at their practice, highlighting the important role pharmacists have in providing adult vaccinations as part of an immunization neighborhood.

Pharmacists play a key role in providing vaccinations to adults. First, some adult vaccines are covered by Medicare Part D, a pharmaceutical benefit, and therefore cannot easily be delivered in a PCP office. Medicare Part B, an office-based benefit, provides coverage for most vaccines that adult patients need, including hepatitis $\mathrm{B}$, influenza, and pneumococcal vaccines and vaccines directly related to treatment of an injury or direct exposure to a disease or condition. Medicare Part D covers all commercially available vaccines, except those covered by Part B. ${ }^{13}$ A recognized challenge in the delivery of adult vaccines is the presence of these various Medicare plans. ${ }^{14}$ There are platforms (TransactRX ${ }^{\odot}$ ) by which PCPs can bill for Medicare Part D covered vaccines, though this process can be cumbersome; therefore, it is often less complicated for patients to receive vaccinations covered by Medicare Part D at a pharmacy. Having all adult vaccines covered by Medicare Part B has been considered but ultimately decided against, ${ }^{15,16}$ thus care needs to be given to better systematically improve the current process. Second, the practice of pharmacists providing adult vaccinations has substantially increased since $1995 .{ }^{17}$ Not only are more US- 
Figure 1. Physicians attitudes and experience regarding delivery of vaccines to adult patients, United States, 2019 (n = 642). Abbreviation: PCP, primary care physician.

- Strongly agree $\quad \square$ Somewhat agree $\quad \square$ Somewhat disagree $\quad \square$ Strongly disagree

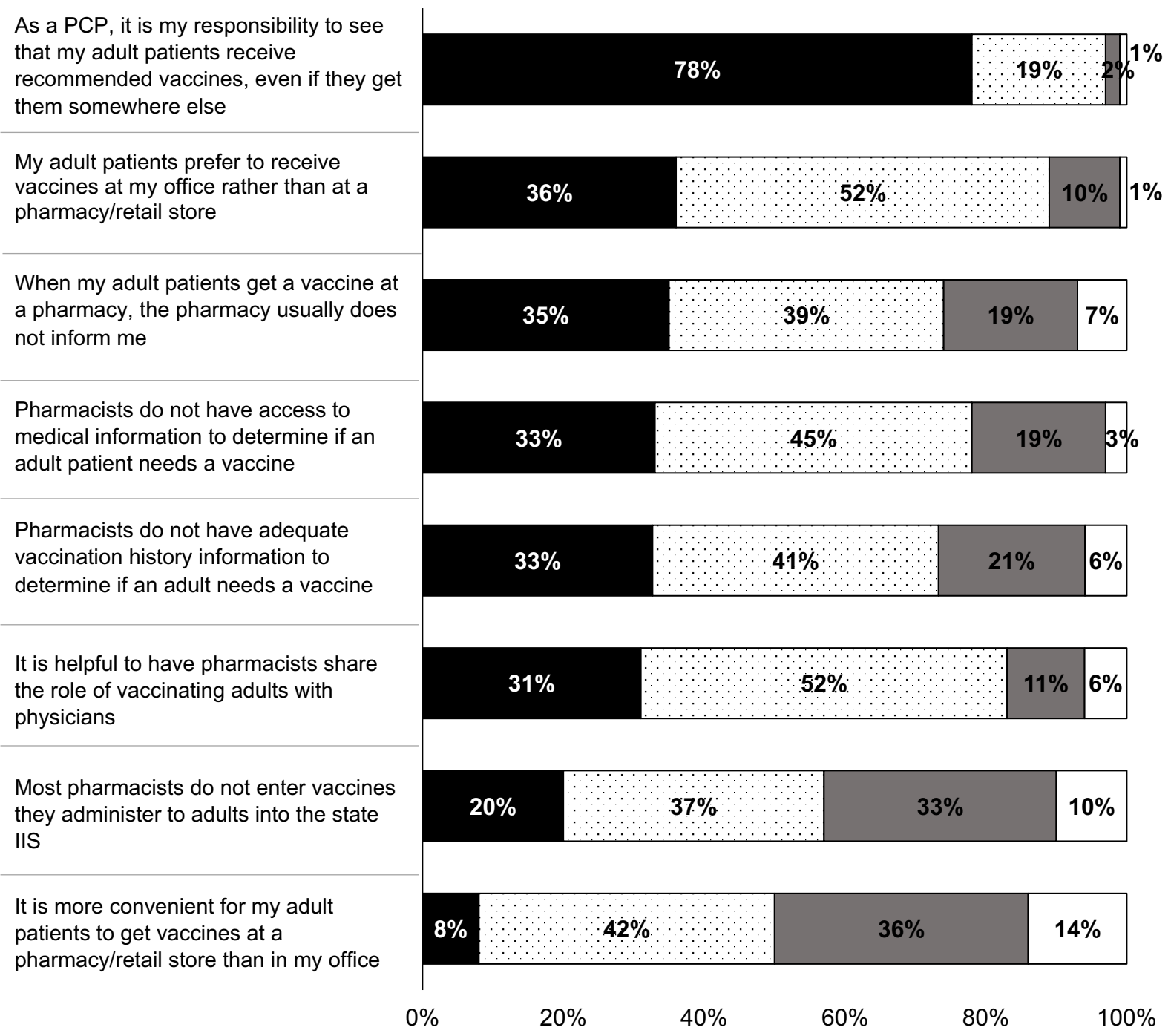

based pharmacy schools incorporating vaccination practices into their curriculum but more than $90 \%$ of the US population lives within 2 miles of a pharmacy, making pharmacy-based immunization services a more convenient way to obtain an important health service. $^{18-20}$

Alongside PCPs' reported concerns about pharmacists giving vaccinations, pharmacists have also reported barriers including lack of time or staff at the pharmacy and inadequate vaccination expertise. ${ }^{21}$ Knowing that both PCPs and pharmacists have concerns and recognizing that pharmacists are an integral part of the immunization neighborhood is important for furthering the pharmacist-provider relationship. These findings and known challenges highlight key opportunities for pharmacists to ensure they are following NVAC published standards and establishing immunization neighborhoods. The NVAC standards state that all vaccinators, pharmacists included, incorporate routine immunization assessment into daily interactions with patients, administer recommended and needed vaccinations, provide patients with vaccine information, record the vaccination in the vaccine registry, and communicate receipt of vaccination with the patient's medical home as well as serve as 
a role model for patients. ${ }^{4,6}$ Pharmacists fulfilling the NVAC standards, specifically recording within the IIS and communicating receipt of vaccination to the patient's medical home, will help to strengthen the immunization neighborhoods that have already been established and would help to address the concerns raised by PCPs.

Documentation of vaccination by pharmacists within their employer's electronic medical record is likely occurring on a consistent basis, but it is not standard practice across the United States that pharmacists are documenting within the IIS. A 2014 survey by the American Immunization Registry Association assessed pharmacy reporting of vaccinations to an IIS. Of the 45 state IISs that responded, 36 (80\%) reported that pharmacies reported vaccinations to the IIS, but only 22 (49\%) states had mandatory reporting laws for pharmacies. ${ }^{22}$ The variation among states as well as not having mandatory reporting laws or a streamlined process for reporting in all states impacts the ability for pharmacists to adequately report receipt of vaccination to an IIS and highlights the need for changes in policies and procedures.

There are both strengths and limitations to our study. The results of this study were generated from PCPs across the nation with an overall excellent response rates for a physician survey. Although the sample of PCPs was designed to be representative of ACP and AAFP memberships, the experience and attitudes reported may not be fully generalizable. In addition, nonrespondents may have had different views or experiences with pharmacists giving vaccinations than respondents did.

To our knowledge, this is the first survey to assess the physician's perspective on the role of pharmacists in delivering vaccinations. These findings illustrate that PCPs have mixed feelings about pharmacists delivering vaccinations to adults. Greater use of the IIS and communication by pharmacists to the patient's medical home could partially address physicians' concerns by providing a systematic way for pharmacists and physicians to share patient vaccination history.

The authors would like to thank Amy Mullins, MD and Bellinda Schoof, MHA from the AAFP and Selam Wubu, MPH and Darilyn Moyer, MD from the ACP for collaborating in the establishment of the sentinel network in family medicine and general internal medicine. We would also like to thank all family physicians and general internists in the networks for participating and responding to this survey.
To see this article online, please go to: http://jabfm.org/content/ 34/2/392.full.

\section{References}

1. Williams WW, Lu PJ, O'Halloran A, et al. Surveillance of vaccination coverage among adult populations-United States, 2015. MMWR Surveill Summ 2017;66:1-28.

2. Centers for Disease Control and Prevention. National and state-level place of flu vaccination among vaccinated adults in the united states, 2014-15 flu season. Available from: https://www.cdc.gov/flu/fluvaxview/ place-vaccination-2014-15.htm. Accessed November 4, 2020.

3. Schmit CD, Penn MS. Expanding state laws and a growing role for pharmacists in vaccination services. J Am Pharm Assoc 2017;57:661-9.

4. Rothholz M, Tan LLJ. Promoting the immunization neighborhood: benefits and challenges of pharmacies as additional locations for HPV vaccination. Hum Vaccin Immunother 2017;13:1856-8.

5. American College of Emergency Physicians. The patient-centered medical home model. Ann Emerg Med 2016;68:147-8.

6. National Vaccine Advisory Committee. Recommendations from the National Vaccine Advisory Committee: standards for adult immunization practice. Public Health Rep 2014;129:115-23.

7. Tanzi MG. It takes a village: NVAC standards emphasize importance of immunization neighborhood. 2014. Available from: pharmacist.com/article/ it-takes-village-nvac-standards-emphasize-importanceimmunization-neighborhood. Accessed August 6, 2020.

8. Crane LA, Daley MF, Barrow J, et al. Sentinel physician networks as a technique for rapid immunization policy surveys. Eval Health Prof 2008;31:43-64.

9. Brtnikova M, Crane LA, Allison MA, Hurley LP, Beaty BL, Kempe A. A method for achieving high response rates in national surveys of U.S. primary care physicians. PLoS One 2018;13:e0202755.

10. McMahon SR, Iwamoto M, Massoudi MS, et al. Comparison of e-mail, fax, and postal surveys of pediatricians. Pediatrics 2003;111:e299.

11. Atkeson LR, Adams AN, Bryant LA, Zilberman L, Saunders KL. Considering mixed mode surveys for questions in political behavior: using the Internet and mail to get quality data at reasonable costs. Polit Behav 2011;33:161-78.

12. Dillman DA, Smyth JD, Christian LM. Internet, phone, mail, and mixed-mode surveys: the tailored design method. Wiley; 2014.

13. Medicare Part D Vaccines. Medicare learning network fact sheet. 2019. Available from: https://www. cms.gov/Outreach-and-Education/Medicare-LearningNetwork-MLN/MLNProducts/Downloads/VaccinesPart-D-Factsheet-ICN908764.pdf. Accessed August 6, 2020. 
14. Hurley LP, Bridges CB, Harpaz R, et al. U.S. physicians' perspective of adult vaccine delivery. Ann Intern Med 2014;160:161.

15. U.S. Government Accountability Office. Medicare: many factors, including administrative challenges, affect access to Part D vaccinations. 2011. Available from: www.gao.gov/products/GAO-12-61. Accessed September 14, 2020.

16. Medicare Payment Advisory Commission. Issues in Medicare coverage of drugs. In: Report to the Congress: promoting greater efficiency in Medicare. 2007:157-86. Available from: www. medpac.gov/chapters/Jun07_Ch07.pdf. Accessed September 14, 2020.

17. Hogue MD, Grabenstein JD, Foster SL, Rothholz MC. Pharmacist involvement with immunizations: a decade of professional advancement. J Am Pharm Assoc 2006;46:168-79; quiz 179-82.

18. McConeghy KW, Wing C. A national examination of pharmacy-based immunization statutes and their association with influenza vaccinations and preventive health. Vaccine 2016;34:3463-8.

19. Prescott WA, Jr., Bernhardi C. Immunization education in US pharmacy colleges and schools. Am J Pharm Educ 2019;83:6765.

20. Qato DM, Zenk S, Wilder J, Harrington R, Gaskin D, Alexander GC. The availability of pharmacies in the United States: 2007-2015. PLoS One 2017;12:e0183172.

21. Srivastav A, Black CL, Lutz CS, et al. U.S. clinicians' and pharmacists' reported barriers to implementation of the standards for adult immunization practice. Vaccine 2018;36:6772-81.

22. American Immunization Registry Association. Survey of immunization reporting to immunization information systems by major US pharmacies: a summary of methods, successes, and challenges of pharmacy-IIS interfaces. 2014. Available from: immregistries.org/resources/AIRA_Pharmcy_IIS_ White_Paper.pdf. Accessed August 20, 2020. 